\title{
A Wearable Computer System with Augmented Reality to Support Terrestrial Navigation
}

\author{
Bruce Thomas ${ }^{1}$, Victor Demczuk ${ }^{2}$, Wayne Piekarski ${ }^{3}$, David Hepworth ${ }^{3}$, and Bernard Gunther ${ }^{1}$ \\ School of Computer and ${ }^{1}$ \\ Information Science \\ University of South Australia \\ Land Operations Division ${ }^{2}$ \\ Defence Science and \\ School of Physics and ${ }^{3}$ \\ The Levels, SA, Australia \\ Technology Organisation \\ Salisbury, SA, Australia \\ Electronic Systems Engineering \\ University of South Australia \\ The Levels, SA, Australia \\ Bruce.Thomas@UniSA.Edu.Au
}

\begin{abstract}
To date augmented realities are typically operated in only a small defined area, in the order of a large room. This paper reports on our investigation into expanding augmented realities to outdoor environments. The project entails providing visual navigation aids to users. A wearable computer system with a see-through display, digital compass, and a differential GPS are used to provide visual cues while performing a standard orienteering task. This paper reports the outcomes of a set of trials using an off the shelf wearable computer, equipped with a custom built navigation software package, "map-in-the-hat."
\end{abstract}

\section{Introduction}

A new physical form of a portable computer has emerged in the form of a wearable computer [2]. Instead of the computer being hand-held, it is attached to the user on a backpack or belt, as illustrated in Figure 1, leaving the hands free when the computer is in use, and also allowing the user to view data in the privacy of a head mounted display (HMD) [7]. The application areas for this form of computer range from factory monitoring, stock taking, field data collection, to soldiers in the field.

In common with other recent research [6], we are investigating the use of a wearable computer with augmented realities in an outdoor environment (WCAROE). We have been working with the Australian Defence Science and Technology Organisation (DSTO) and the Australian Army investigating ways of improving the effectiveness of the dismounted combat soldier, for which navigation is a significant task.

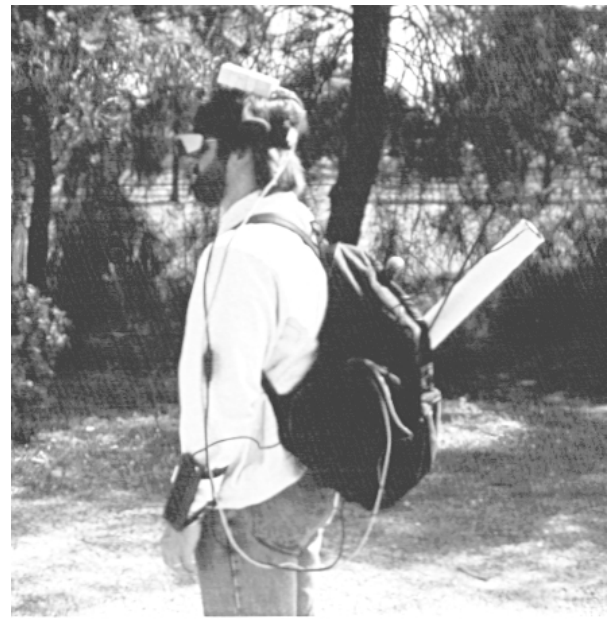

Figure 1: Backpack version of the wearable computer

The application we are investigating is the use of a WCAROE to support navigational tasks, or as we like to call it, "map-in-the-hat." We are proposing to extend the use of GPS with an augmented reality user interface. A key objective of our work is extending augmented reality systems from room size areas to outdoor environments [1].

\section{Related Work}

Augment reality has been used for Heads Up Displays for aviation, assistance for surgery [8] and maintenance work [4]. This augmented reality work can be characterised as requiring precise tracking in small operating regions.

The work presented in this paper breaks from the requirement of precise tracking in small operating

In ISWC1998, 2nd International Symposium on Wearable Computers

October, 1998 - Pittsburgh, PA, USA - Copyright (C) 1998 IEEE

Please visit http://www.tinmith.net for more information 
regions. A few researchers are investigating large area augmented reality. Most notable is the work of Steven Feiner et.al. with Touring Machine project. The Touring Machine allows uses to walk around the Columbia campus and access information via a tracked see-through display and a hand-held display. The three main themes of the their work are as follows: 1) presenting University contextual information visually connected to the physical world, 2) supporting a relatively large area in which the user is able to walk around in, and 3) combining multiple display and interaction technologies. The work presented in this paper extends this concept to a new application, terrestrial navigation.

\section{Navigation Task}

Navigation is the process which guides movement between two points, and enables the navigator to know exactly where they are at any given time. Navigation involves position finding, direction finding and measuring distance. Position finding is typically done by reference to a map - a scaled plan of a portion of the earth's surface. The direction is usually assessed by use of a magnetic compass. When planning a route, the distances to be travelled between waypoints are determined in case position finding is prevented due to poor feature visibility. Distance can be measured by pacing or time calculations.

A typical navigation task is to navigate from point A to point B through a set of waypoints W1...Wn. The user starts at position A and initially navigates to position $\mathrm{W} 1$. Once at waypoint $\mathrm{W} 1$, the user then navigates to position $\mathrm{W} 2$. This process continues until the final position $\mathrm{B}$ is reached.

\subsection{Problems with Traditional Naviga- tion}

Navigation using the above means can be quite difficult and requires considerable training and concentration. Time and attention spent on navigation means less attention is paid to the task environment. There are many environments where manual navigation is difficult and error prone. The military attempt to cope with this by sharing the navigation task amongst the team and by training and practice. A novel use of Virtual Reality is for terrain familiarisation in natural environments [3]; where the user can be trained for a particular set of waypoints.

\subsection{Global Positioning System}

With GPS, a navigator applies a similar technique to that traditionally used, except for the addition of accurate positioning information. The user knows within the accuracy of the GPS their current location, thereby greatly enhancing their ability to plot their current position on a map.

\section{The Wearable Computer System}

The core of the wearable computer system used in our experiments is the Phoenix 2 wearable computer [5]. This system has been used in previous experiments described in $[9,10]$, involving test on input devices for wearable computers.

With our current research, the computer system has become a lot more complex, with extra peripherals and more batteries, making it impractical to mount everything comfortably on a belt. Therefore, a backpack was used to carry all the needed components, see Figure 1. The central computer is Phoenix 2 Wearable Computer (with a Cyrix 486DX2/66 Processor, 32 MB RAM, and a 850 MB 2.5" hard disk drive.) The operating system is Slackware Linux v3.3 running kernel version 2.0.30. The GPS system is a Trimble SVeeSix-CM3 GPS Core Module (NMEA-0183 output and RTCM-104 input), a Aztec RDS3000 Differential GPS Receiver Module (RTCM-104 output.) The electronic compass is Precision Navigation TCM2. The Sony PLM-100 dual colour personal LCD monitor is the see-through device. A VGA to NTSC converter board is needed since the Sony display requires a NTSC signal.

\subsection{Software Operation}

The main purpose of the software is to use the output from the hardware devices, such as the GPS and compass, to produce navigation information which is displayed to the user, as in Figure 2.

To use our system, the user must first enter a list of waypoints in the form of WGS84 latitude and longitude coordinate points. Once the waypoints have been entered, the user starts the map-in-the-hat program. Keypresses scroll through the current waypoint displayed on the screen.

The compass at the top of the screen in Figure 2 shows where the viewer's head is currently pointed, the small triangle underneath with the value indicates 


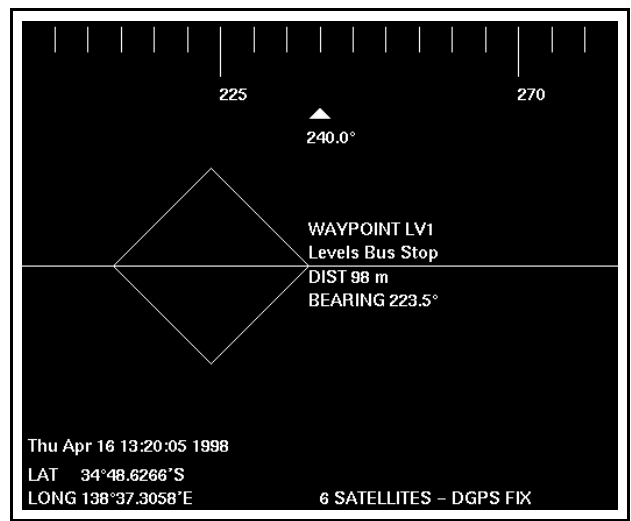

Figure 2: User's screen view

the exact direction in degrees true ${ }^{1}$. The diamond on the display represents the waypoint the user is heading towards, and is positioned relative to the compass at the top of the screen; it is meant to approximate where the object would be positioned in the physical world. However, perspective corrections have not been implemented yet and so the diamond will only overlay the target exactly when the user is looking straight at it. To the right of the diamond is information showing the name of the waypoint, and its distance and bearing. When the user is within 1000 meters of the next waypoint, the size of the diamond cursor increases as the user approaches the waypoint.

To reach the target, the user looks through the display and attempts to walk to where the diamond is located. If the diamond is to the left of the display centre, then the user rotates their head to the left until the diamond is centred on the screen. If the direction to the next waypoint is outside the field of view, the diamond cursor is placed on the side of the screen and filled with hash marks. The side of the screen the cursor is placed on indicates the direction for the user to turn. The display works in a similar way to the head up display used on military aircraft, where a box is placed around the target on the display, and the pilot can fly the plane toward the target by keeping it in the centre of the glass.

The bottom left portion of the display shows the current date and time. Under this information is the user's position as a WGS84 latitude and longitude coordinate. The bottom right portion of the display

\footnotetext{
${ }^{1}$ True North is the direction of the shortest global arc that intersects with the axis of the earth's rotation, and Magnetic North points in the direction of the horizontal component of the earth's magnetic field at its current location, and not actually to True North.
}

shows the number of GPS satellites in contact with the receiver.

\section{Trials}

We performed three field trials of our navigation system to test the system in a realistic outdoor setting. The trials tested three different conditions: waypoints a large distance apart, a large set of waypoints, and waypoints in an urban setting. The limitations of the SONY display power consumption forced us to limit the trials to no longer than 2.5 hours each.

\subsection{Trial 1 - Long Distances}

The first trial was between waypoints a large distance apart with obstacles between them, such as buildings to force us to deviate from the prescribed bearing. This trial included waypoints roughly a distance of 1.7 kilometres maximum from the starting position. Because the display has a battery life of only 2.5 hours, this trial only tested a start, middle and end waypoint.

The navigation system provided accurate bearing and distance information to all three waypoints. Deviations while walking were needed to be undertaken to avoid construction works, and the navigation system behaving accordingly. The accuracy obtained was to 5 to 20 metres. A problem we noted was that sometimes the GPS signal would completely disappear in open terrain with no discernible features blocking the signal, but after a moment or two the signal was reacquired.

\subsection{Trial 2 - Large Data Set}

The second trail used six waypoints, derived from survey markers and within a kilometre of each other. The first three waypoints were within a 30 metre radius of each other, to test the system's accuracy. Unfortunately, only 20 metre accuracy was achieved. These points were under a set of high-tension powerlines, and this may have been the cause of the poor accuracy, as the differential GPS signal was carried on a radio signal.

On the final three waypoints, about 400 meters apart, we were able to achieve an accuracy of metres for two of them and 17 metres for the other. Light tree cover on occasions interfered with the GPS signal, and like the previous trial, the signal was lost for a short period of time out in the open with no blocking terrain. 


\subsection{Trial 3 - Urban Setting}

This trial was performed with given waypoints we had not seen before. A series of five waypoints were visited, all within 200 metres of each other and in a city environ, to assess how the GPS would perform in an urban setting. This was not expected to be easy for the GPS.

Initially, no GPS signal was procured due to the buildings and trees prevalent in the city, but three satellites were obtained with a DGPS fix by standing in an open parking lot. We followed the bearing to the first waypoint, to within 5 metres. The next point was found to within 10 metres accuracy.

Waypoint three was much the same as waypoint two; the diamond cursor jumped considerably within the final 20 metre radius. Attempts were made to reduce the distance to the waypoint by not following the bearing when closer than 20 metres, but rather by finding the closest distance measurement. The result was an accuracy of 5 metres. Journeying to waypoint 5 , the final test for the day, produced an accuracy of 2 metres.

\section{Conclusion}

With our system, we have demonstrated a hands free navigational aid to a person navigating on foot. The use of a wearable computer system with a seethrough head mounted display provided a functional platform to develop our system. Differential GPS proved to be effective for locating one's position to 20 metre and better accuracy.

The map-in-the-hat application provided useful visual cues for the navigation task. Further work is needed to better understand the form and content of the presented information to optimise visual cues. It is a delicate balance that must be maintained between presenting enough information and obscuring the user's view of the physical world.

A number of observations were made. Firstly, the digital compass is susceptible to vibrations while walking on rough terrain. Secondly, the see-through display has a control to adjust the back lighting of the screen (this adjusts how opaque the screen becomes). which turned out to be a very useful feature in changing light conditions. However, excessive sunlight is a problem, and obscures the SONY display. Thirdly, increasing the size of the diamond cursor proved to be a subtle but effective cue in determining when the user is becoming closer to a waypoint. Finally as with most wearable computer systems, the power management proved to be a major concern of our system.

\section{References}

[1] Ronald T. Azuma. Survey of augmented reality. Presence: Teleoperators and Virtual Environments, 6(4), August 1997. To be published.

[2] Len Bass, Chris Kasabach, Richard Martin, Dan Siewiorek, Asim Smailagic, and John Stivoric. The design of a wearable computer. In $A C M C H I$ 97, pages 139-146, March 1997.

[3] R.P. Darken and W.P Banker. Navigating in natural environments: A virtual environment training transfer study. In Proc. of VRAIS '98, pages $12-19,1998$.

[4] Samory Karez, Vania Conan, and Pascal Bisson. Virtually documented environments. In Proc. of ISWC '97, pages 158-160. IEEE, 1997.

[5] Inc. Phoenix Group. Phoenix 2 Wearable Computer. 123 Marcus Blvd., Hauppauge, New York 11788, USA. http://ivpgi.com/.

[6] S. Feiner, B. MacIntyre, T. Höllerer and A. Webster. A touring machine: Prototyping 3D mobile augmented reality systems for exploring the urban environment. In Proc. of $I S W C$ '97, pages 74-81. IEEE, October 1997.

[7] T. Starner, S. Mann, B. Rhodes, J. Healey, K. B. Russell, J. Levine, and A. Pentland. Wearable computing and augmented reality. Technical Report 355, M.I.T. Media Lab, November 1995.

[8] A. State, M. Livingston, W. Garrett, G. Hirota, M. Whitton, E. Pisano, and H. Fuchs. Technologies for augmented reality systems: Realizing ultrasound-guided needle biopes. In Proc. SIGGRAPH '96, pages 439-446, August 1996.

[9] B. H. Thomas, S. P. Tyerman, and K. Grimmer. Evaluation of three input mechanisms for wearable computers. In Proc. of ISWC '97, pages 2-9. IEEE, 1997.

[10] S. Tyerman and B. Thomas. Functionality and usability of novel input devices on a wearable computer. In ACSC'98 - The 21st Australasian Computer Science Conference, pages 205-216, January 1998. 